\title{
Modelling and Sampling Ramified Objects with Substructure-Based Method
}

\author{
Weiwei Yin ${ }^{1}$, Marc Jaeger ${ }^{2}$, Jun Teng ${ }^{1}$, \\ and Bao-Gang $\mathrm{Hu}^{1}$ \\ 1 Institute of Automation, \\ Chinese Academy of Sciences, China \\ 2 CIRAD, AMAP, France \\ \{wwyin, jaeger, jteng, hubg\}@liama.ia.ac.cn \\ http://liama.ia.ac.cn
}

\begin{abstract}
This paper describes a technique that speeds up both the modelling and the sampling processes for a ramified object. By introducing the notion of substructure, we divide the ramified object into a set of ordered substructures, among which only a part of basic substructures is selected for implicit modelling and point sampling. Other substructures or even the whole object can then be directly instantiated and sampled by simple transformation and replication without resorting to the repetitive modelling and sampling processes.
\end{abstract}

\section{Introduction}

Smooth blending junctions and complex structures are two distinct characters for many ramified objects. Some parametric approaches [1] [9] have been explored to model and visualize such objects. While, implicit methods [2] which we would prefer more, well exhibit the local details in ramification due to their unmatched advantages in generating smooth blending surface.

However, implicit surface has its own difficulty in generating sampling points and surface reconstruction when rendering. Bloomenthal and Wyvill first proposed to use scattered seed points to sample implicit surface in [2]. After that, more attentions were paid on the physically based sampling algorithms in [4] [5]. Witkin implemented a quite complete particle system to sample blobby spheres and cylinders in [6].

In this paper, we do aim to efficiently model and sample such ramified object with smooth blending junctions. With the precondition that the ramified object can be hierarchically organized, we recursively divide the object into a set of ordered substructures, among which only a part of basic substructures is selected as the new target for implicitly modelling and basic point sampling. Other substructures or even the whole object can then be directly instantiated and sampled by transforming and replicating sampling points of the new target without real geometric modelling and sampling processes. 


\section{Object Decomposition}

We consider a ramified object consisting of a main branch and several lateral branches, which may have other lateral branches on them. Based on the botanical knowledge, we separate the lateral branch from the main axis and divide this ramified object into several types of similar subparts (substructures) 7]. Each substructure is assigned a hierarchical order and redivided in the same way until it reaches the basic form $S u b_{b a s i c}$ as:

$$
\text { Sub }_{\text {basic }}=\left\{\text { Axis }+ \text { Skeleton }_{0}+\cdots+\text { Skeleton }_{i}+\cdots+\text { Skeleton }_{m}\right\}
$$

where Skeleton $_{i}$ denotes a single lateral branch attached to the main axis Axis and can not be redivided.

An example of two-step decomposition for a ramified object is shown in the following equations:

$$
\begin{aligned}
& \text { Ojbect }=\left\{\text { Axis }_{\text {main }}+S u b_{1}+\cdots+S u b_{i}+\cdots+S u b_{n}\right\} \\
& S u b_{i}=\left\{\text { Axis }_{\text {imain }_{\text {man }}}+S u b_{i 1}+\cdots+S u b_{i j}+\cdots+S u b_{i k}\right\}
\end{aligned}
$$

where $A x i s_{\text {main }}$ and $A x i s_{i_{\text {main }}}$, represented by a single branch, denote the corresponding main branches, $S u b_{i}$ denotes the substructure in hierarchy 1, $S u b_{i j}$ denotes the substructures in hierarchy 2 that constitute $S u b_{i}$ in hierarchy 1.

Since our decomposition is fully based on the connective relationship between the main axis and the lateral branch, a neighborhood graph for substructures either of the upper-lower hierarchies or among the same hierarchy can be easily built up. Additionally, level of detail (LOD) techniques can be conveniently applied in such defined system because all substructures in each order can be quickly reached and retrieved through the neighborhood graph.

\section{$3 \quad$ Implicit Modelling}

A common and intuitive method for modelling smoothly blending surface is based on the underlying skeletons [2] 3. A skeleton-based implicit surface is defined as a set of points $P(x, y, z)$ as follows:

$$
\text { Surface }=\left\{P(x, y, z) \in \Re^{3} \mid f(P)=0\right\}
$$

where $f(x, y, z)$ is a field function defined by the skeleton.

For a branching structure consisting of skeletons $s_{i}(i=1, \cdots, n)$ with the associated field functions $f_{i}(i=1, \cdots, n)$, we define such a skeleton-based implicit surface and apply blending techniques, for instance the simplest sum operation $f(P)=\sum_{i=1}^{n} f_{i}(P)$, to generate smooth blending surface. 


\section{Point Sampling}

The basic idea of point sampling is inspired by Witkin [6], who generated a well distributed sample of particles while had high computational cost when the sampling particle number was huge. In order to improve sampling efficiency as well as remain the high sampling density, we propose a substructure-based method, which uses a part of the ramified object instead of the whole one as the sampling target. Since the target is shrunk and simplified, the sampling particle number is reduced, followed by the computational complexity.

Six steps are included in our substructure-based sampling method and each step is briefly described in the following:

Step 1. Define a new target by selecting a representation from each type of basic substructures.

Step 2. Model the new target with skeleton-based implicit surfaces.

Step 3. Apply Witkin's sampling algorithm [6] to the implicitly modelled surfaces of the new target. Relevant information of sampled particles, including 3D positions and surface normals, is stored.

Step 4. Instantiate other substructures and the whole object by directly involving and linear geometric transforming the sampled points of the new target.

Step 5. Detect ramiform particles which come from different substructures and penetrate each other as shown in Fig 1 (left). A particle $P(x, y, z)$ is considered as a ramiform particle only when

- It is inside the ramification area between substructures, and

- The field function value at it satisfies: $|f(P)|>\varepsilon$ where $\varepsilon$ is a very small positive number.

Step 6. Delete all ramiform particles and apply Witkin's sampling algorithm again to the skeletons which lie inside the corresponding ramification. A new blending surface will be quickly generated as shown in Fig 1 (left-center).

Non-ramiform particles are considered static on their own position. Sampling time and computational cost are greatly reduced without managing all particles at each iteration even if the total number of the particles is very huge. Moreover, since only ramifications existing between upper-lower substructures are considered, no unwanted blending will exist between actually non-connected branches.

\section{Implementation and Results}

The techniques described in this paper have been implemented in $\mathrm{C}++$ code. The groundwork of our present experiments is the GreenLab Model [8] whose output is a set of hierarchically structured line skeletons. A simple exponential function is defined as the field function $f_{i}$ for the $i$ th line skeleton:

$$
f_{i}(P)=\exp \left[-\frac{d_{i}(P)^{2}}{R_{i}^{2}}+1\right]-1
$$


where $d_{i}(P)$ is the algebraic distance of point $P(x, y, z)$ to the $i$ th skeleton. $R_{i}$, a radius parameter, may be a constant for a constant-radius branch or be computed by certain linear variable function for a tapered branch.

Some simple examples are shown below. A substructure composed of two kinds of basic forms is shown in Fig 1 (center). Moreover, with the original one shown in Fig 1 (right-center), the result of adding a new branch in Fig 1 (right) is quickly achieved with no need to sample all the skeletons again.
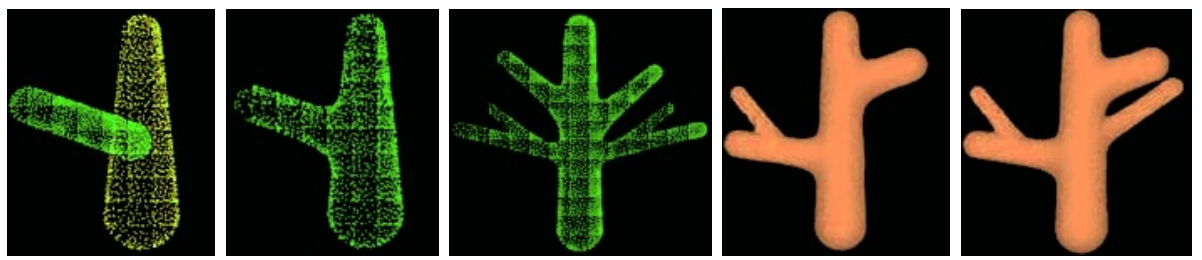

Fig. 1. Left-Right: Before merging (left); After merging (left-center); An example of substructures (center); Before adding a new branch (right-center); After adding a new branch (right)

\section{Conclusions and Future Work}

In this work, we have proposed a substructure-based method to implicitly model and sample ramified objects. With the shrunk and simplified target and the utilization of substructures, both the modelling and sampling processes are sped up and repetitious works for modelling and sampling the same type of substructures are avoided. As for the future works, the proposed method will be applied to real ramified objects. A texture algorithm for sampling particles is also required.

\section{Acknowledgement}

This work is supported in part by the national Natural Science Foundation of China (\#60073007, \#60473110) and by China 863 Program (\#2002AA241221).

\section{References}

1. Bloomenthal, J.: Modeling the Mighty Maple. In: Proc.SIGGRAPH'85, Vol. 19. (1985) 305-311

2. Bloomenthal, J., Wyvill, B.: Interative Techniques for Implicit Modeling. In: Computer Graphics Forum, Vol. 24. (1990) 109-116

3. Bloomenthal, J., Shoemake, K.: Convolution Surfaces. In: Proc.SIGGRAPH'91, Vol. 25. (1991) 251-256

4. Turk, G.: Generating Textures on Arbitrary Surfaces Using Reaction-Diffusion. In: ACM TOG, Vol. 25. (1991) 289-298

5. de Figueiredo, L.H., Gomes, J.: Sampling Implicit Objects with Physically-Based Particle Systems. In: Computer Graphics, Vol. 20. (1996) 365-376 
6. Witkin, A.P., Heckbert, P.S.: Using Particles to Sample and Control Implicit Surfaces. In: Proc.SIGGRAPH'94. (1994) 269-277

7. Yan, H.P., Barczi, J.F., de Reffye, P., Hu, B.-G.: Fast Algorithms of Plant Computation Based on Substructure Instances. In: Proc.the 10th International Conference in Central Europe on Computer Graphics'02, Vol, 10. (2002) 145-153

8. Yan, H.P., Kang, M.Z., de Reffye, P., Dingkuhn, M.: A Dynamic Architectural Plant Model Simulating Resource-Dependent Growth. In: Annals of Botany, Vol, 1. (2004) 591-602

9. Felkel, P., Kanitsar, A., Fuhrmann, A., Wegenkittl, R.: Surface Models of Tube Trees. In: Computer Graphics International'04. (2004) 70-77 